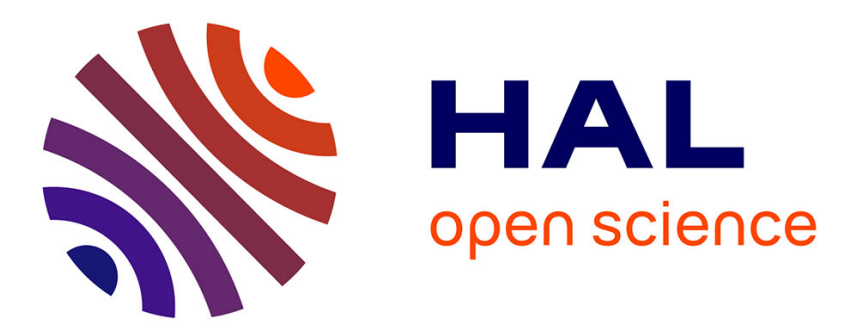

\title{
Modal instability suppression in a high-average-power and high-finesse Fabry-Perot cavity
}

Loïc Amoudry, Huan Wang, Kevin Cassou, Ronic Chiche, Kevin Dupraz, Aurélien Martens, Daniele Nutarelli, Viktor Soskov, Fabian Zomer

\section{- To cite this version:}

Loïc Amoudry, Huan Wang, Kevin Cassou, Ronic Chiche, Kevin Dupraz, et al.. Modal instability suppression in a high-average-power and high-finesse Fabry-Perot cavity. Appl.Opt., 2020, 59 (1), pp.116-121. 10.1364/AO.59.000116 . hal-02475205

\section{HAL Id: hal-02475205 https://hal.science/hal-02475205}

Submitted on 26 Nov 2020

HAL is a multi-disciplinary open access archive for the deposit and dissemination of scientific research documents, whether they are published or not. The documents may come from teaching and research institutions in France or abroad, or from public or private research centers.
L'archive ouverte pluridisciplinaire HAL, est destinée au dépôt et à la diffusion de documents scientifiques de niveau recherche, publiés ou non, émanant des établissements d'enseignement et de recherche français ou étrangers, des laboratoires publics ou privés. 


\title{
Modal instabilities suppression in high average power and high finesse Fabry-Perot Cavity
}

\author{
Loïc Amoudry ${ }^{1}$, Huan Wang ${ }^{1}$, Kevin Cassou ${ }^{1}$, Ronic Chiche ${ }^{1}, \mathrm{KeVin} \mathrm{Dupraz}^{1}$, Aurélien \\ Martens $^{1}$, Daniele Nutarelli ${ }^{1}$, VikTor SOSKov ${ }^{1}$, and Fabian ZOMER ${ }^{1}$ \\ ${ }^{1}$ LAL, Univ. Paris-Sud, CNRS/IN2P3, Université Paris-Saclay, 91898 Orsay, France \\ ${ }^{*}$ Corresponding author: amoudry@lal.in2p3.fr
}

Compiled November 4, 2019

\begin{abstract}
An experimental method to remove modal instabilities induced by thermoelastic deformation in optical high finesse resonators is presented and experimentally investigated. The method is found suitable for multi-mirror folded monolithic and compact cavities as those used in particle accelerator environment. It is also suitable for very large stacked average power. Stable operation at $200 \mathrm{~kW}$ intracavity average power is experimentally demonstrated. @ 2019 Optical Society of America
\end{abstract}

http://dx.doi.org/10.1364/ao.XX.XXXXXX

\section{INTRODUCTION}

External Fabry-Perot cavities (FPC) sustaining resonant highaverage laser-power are nowadays foreseen for various applications. In pulsed regime, Compton scattering of laser beams off charged particle beams allows to produce quasi-monochromatic $X$ and $\gamma$ ray beams [1-3]. Interaction of laser beams with gas provides high harmonics in the XUV range [4]. In the context of the latter application an average power of $670 \mathrm{~kW}$ of a 10 picosecond laser beam was reached in a few thousand finesse cavity [5]. In continuous wave regime the LIGO FPCs have been operated with an intracavity average power around $100 \mathrm{~kW}$ $[6,7]$ and an upgrade to $800 \mathrm{~kW}$ is foreseen for the LIGO-VIRGO collaboration. Recently, a research and development program has been launched to reach the challenging goal of $3 \mathrm{MW}$ intracavity power for the photo-neutralization of deuterium in the context of future fusion energy experiments [8].

However, all these applications face the same experimental issue related to thermal effects induced by high average power. One of the most limiting effect appears to be the transverse mode degeneracy $[5,9]$ induced by thermo-elastic deformation of the mirror surfaces [10,11]. Various methods have been proposed to circumvent this issue. Firstly, the higher order modes resonances can be mitigated by a specific design of the mirror shapes or by additionally heating the mirrors [12-15]. Secondly, when interested in the FPC fundamental modes, losses can be induced to high order modes by means of an intracavity pinhole [16]. Thirdly, effects of thermoelastic deformations can be compensated by inserting a Brewster plate in the FPC [17]. Lastly, for instance in the context of Compton experiments, FPCs have been operated in burst mode making use of the linear accelerator duty cycle which reduces the laser average power to the $\mathrm{kW}$ level at most $[18,19]$.
In this article, we concentrate on FPCs dedicated to Compton $X$-ray sources based on electron rings [3,20]. A few peculiarities concerning the design of these FPCs should be highlighted. They are only accessible with difficulties, and their footprint must be reduced since they are located inside the ultra-high vacuum (UHV) chamber of an electron ring, e.g. below $1 \times 10^{-9} \mathrm{mbar}$ [20]. The use of the fundamental mode with a very small waist, typically few tens of microns, is requested to optimize the X-ray production rate. A very high finesse optical cavity, typically above 20000 is of major interest to reduce the average power delivered by the laser amplifier in a cost reduction strategy. The mechanical design must be stable while providing enough flexibility to adjust the cavity round-trip length, which leads to the choice of four-mirror geometries [21, 22].

Together with the small waist requirement this later constraint implies that the cavity mode can be strongly elliptical $[23,24]$ and operated close to instability region [25]. Eventually, in order to maximize the X-ray flux produced by laser beam scattering off the electron beam one must reduce as much as possible the crossing angle between the electron and laser beams propagation axes. This means that small mirror diameters must be favored since they are imposing a minimum crossing angle value [23].

The list of above technical solutions used to mitigate thermal effects would be extremely difficult to implement or would difficultly fit within the constraints of Compton $X$ ray sources application summarized in the previous section. First, heating the cavity mirrors $[12,13]$ would be very difficult to implement in an electron accelerator environment and not straightforward to apply to one-inch mirror diameters. Shaping the mirrors [14, 15] while keeping a very high reflectivity and thus the highest grade of polishing quality would require dedicated developments on 
mirror technology. Moreover, these methods would have to be adapted to a cavity regime close to the instability where thermoelastic deformations induce very large changes of the elliptical mode radii on mirror surfaces [5]. The pinhole solution [16] does not fit with an elliptical beam shape and could also be damaged when high energy is stacked inside the cavity. Inserting a Brewster plate supplemented by a thermal control as in [17] would be difficult to implement in the UHV and confined electron accelerator environment. In this article we propose a simple solution adapted from [16] that accounts for elliptical mode shapes and, high-finesse, high intracavity power, low loss FPCs. Instead of a pinhole, a pair of movable D-shape dielectric mirrors are used to induce large losses on high-order transverse modes while preserving the high FPC finesse for the fundamental mode. D-shape mirrors with metallic coatings have already been used successfully in [26] to pick-up a small fraction of a short pulse intracavity laser beam in order to perform in situ spectrum measurement.

In this article, we report on the experimental results obtained with this technique. The article is organized as follow. The section 2 is devoted to description of the setup and the model used to describe the effects of thermal heating. The experimental observation of degenerate modes is given in section 3. Finally, significant improvements on the long term operation provided by the implemented solution are put forward in section 4 .

\section{EXPERIMENTAL SET-UP AND MODELING OF HEAT- ING EFFECTS}

Planar bow-tie cavities with four high reflectivity mirrors $\left\{M_{i}\right\}_{i=1, . ., 4}$ are chosen for stability reasons [21,27]. A schematic of the research and development cavity used in the experiments described in this paper is given in Fig 1 . The injection mirror $M_{1}$ is planar and its substrate is made of Suprasil[ [28]. The three other mirror substrates are made of Ultra Low Expansion glass (ULE) which has been shown to be optimum in the high average power regime [5]. All the mirror coatings are made with alternate dielectric layers of $\mathrm{Ta}_{2} \mathrm{O}_{5}$ and $\mathrm{SiO}_{2}$ of $\lambda / 4$ thickness for an incidence angle of $4^{\circ}$. Mirrors have been manufactured and characterized by "Laboratoire des Matériaux Avancés" (LMA) in Lyon (France). $M_{2}$ is planar and mirrors $M_{3}$ and $M_{4}$ are spherical with $50 \mathrm{~cm}$ of radius of curvature. The four-mirror cavity is located inside a vacuum chamber. A cavity finesse of 20000 has been measured [29] for the experiments presented in this article. The cavity modes exhibit two waists, one at mid-distance between $M_{1}$ and $M_{2}$ and the other one between $M_{3}$ and $M_{4}$. The size of the latter can be easily changed by tuning the distance between the spherical mirrors $M_{3}$ and $M_{4}$. The required very small waist and the large beam on the mirrors implies that the cavity is at the limit of the stability. The cavity is seeded by a pulsed laser oscillator at a $1031 \mathrm{~nm}$ wavelength and $133.33 \mathrm{MHz}$ repetition frequency. The pulses are stretched to $250 \mathrm{ps}$ in order to be amplified at a maximum of $40 \mathrm{~W}$ by a fiber amplifier and then compressed down to 68 ps [30]. The laser frequency is locked on the cavity round-trip frequency using the Pound-Drever-Hall method [31, 32].

Even if the mirror coatings absorption is low, about 2 ppm as measured after the coating process, the total absorbed power may be of the order of $1 \mathrm{~W}$ or more due to the high intracavity power of several hundreds of $\mathrm{kW}$. As all the system is under vacuum and the mirror substrate made of ULE, the heat dissipation is not efficient and thermal effects may be very important depending on the cavity geometry.

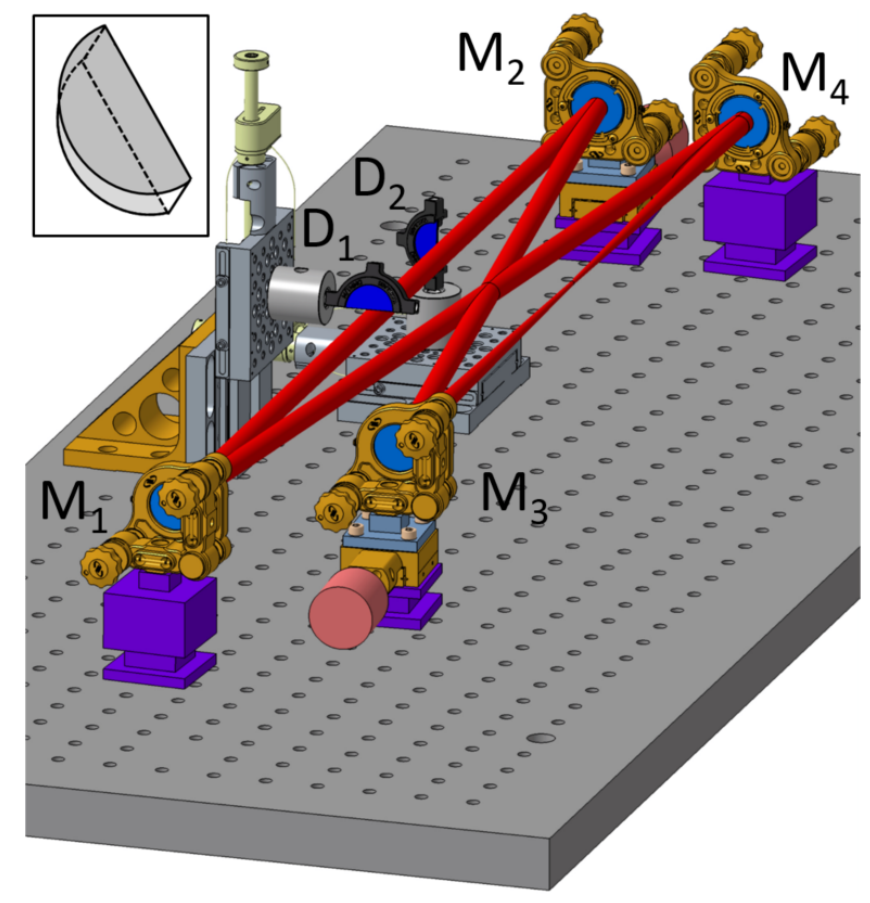

Fig. 1. 3D view of the Fabry-Perot cavity. $M_{1}$ input planar mirror, $M_{2}$ high reflectivity planar mirror, $M_{3}$ and $M_{4}$ high reflectivity spherical mirrors. $D_{1}$ and $D_{2}$ are two half round mirrors, so called D-shaped pickoff mirrors.

In order to model the effect of thermal deformation of the mirror surfaces, one uses the ABCD matrix formalism [25] and a simplified thermo-mechanical model [11]. At the first order of the approximation, the local value of the radius of curvature $R^{\prime}$, given by the absorbed power in the coating at the center of the mirror, can be written as follows

$$
\frac{1}{R^{\prime}}=\frac{1}{R}-\frac{2 \alpha A_{b} P_{l}}{4 \pi \kappa w^{2}}
$$

where $R$ is the initial radius of curvature of the mirror surface, $\alpha$ is the substrate's thermal expansion coefficient, respectively $0.01 \times 10^{-6} \mathrm{~K}^{-1}$ for ULE and $0.6 \times 10^{-6} \mathrm{~K}^{-1}$ for Suprasil, $A_{b}$ is the coating's absorption coefficient, $\kappa$ is the substrate's thermal conductivity, respectively $1.31 \mathrm{~W} \mathrm{~m}^{-1} \mathrm{~K}^{-1}$ for ULE and $1.38 \mathrm{~W} \mathrm{~m}^{-1} \mathrm{~K}^{-1}$ for Suprasil, $P_{l}$ is the intracavity laser power and $w$ is the radius of the laser-beam fundamental mode on the mirror surfaces. For numerical applications we shall use values of $A_{b}=1.2 \mathrm{ppm}$ coming from measurements made after the coating process. It is measured by a mirage effect set-up [33] at factory by the LMA. Measurement of low absorption coefficient in the ppm range is very difficult, the accuracy of the measurement can be estimated to $10 \%$ roughly. The value of $w$ on the mirror surface, given by calculation, is of the order of $1.2 \mathrm{~mm}$.

As the elements of the ABCD cavity round-trip matrix depend on the radius of curvature of the mirrors surface, thermal effects (i.e. intracavity laser power) change their values and impact the stability condition of the cavity, $-1<(A+D) / 2<1$, where $(A+D) / 2$ is the stability parameter. The resonance condition for higher order modes $(\mathrm{q}, \mathrm{n}, \mathrm{m})$ is given in [34]. Due to thermal deformation of the mirror surfaces, higher order transverse modes $T E M_{n m}$ can be resonant at the same time as the fundamental $T E M_{00}$ mode. This resonance degeneracy has been computed as a function of the intracavity laser power and the 
results are shown in Fig. 2. It is observed that for the FPC geometry that we implemented, degenerated resonances are very dense and the order of the degenerated mode vary rapidly with the laser power. Since these high order modes are resonant with the fundamental mode, the energy can be transferred from one to the other and thus generate instabilities. This model has proved to be reliable and efficient for this purpose $[9,11]$.

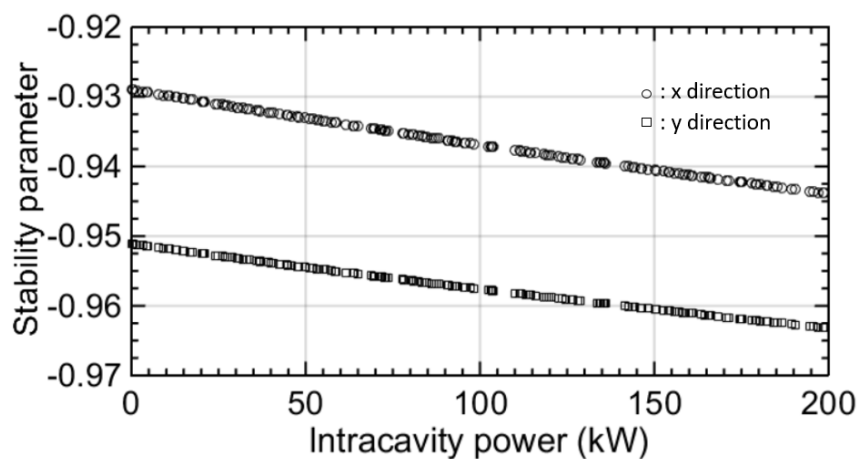

Fig. 2. Stability parameter as function of the intracavity laser power in the cavity. Each circle or square represents the occurrence of a degeneracy between the fundamental and a high order mode $\mathrm{n}$ and $\mathrm{m}$, with $n$ and $m$ ranging from 1 to 30 for both $x$ and $y$ directions.

It is found that the degenerate mode order is quite high in both directions for an intracavity power corresponding to our operating condition as shown in Fig. 3. Furthermore, the extension of a Hermite-Gauss mode of order $n$ in one of the transverse directions is asymptotically given by $\sqrt{n} w$ for large $n$ [35]. It is then possible to introduce strong losses for these modes while preserving the fundamental one by simply using a mask [16].

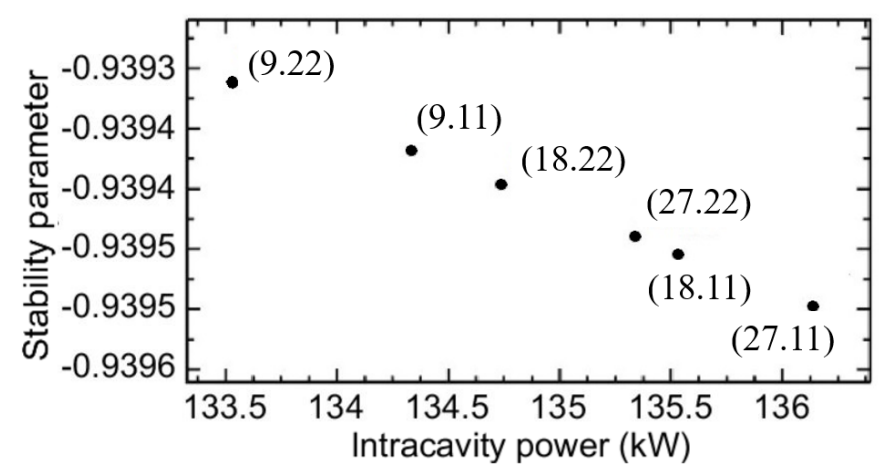

Fig. 3. Zoom on Figure 2 around $135 \mathrm{~kW}$ intracavity power for $x$ direction. The transverse mode numbers are specified on the figure.

\section{EXPERIMENTAL OBSERVATION OF MODAL INSTA- BILITIES}

As shown in Fig. 4 high order modes have been observed in a range of power which are in a good agreement with simulations. For instance, the mode (27.11) has been observed at $110 \mathrm{~kW}$ intracavity power instead of $136 \mathrm{~kW}$, as predicted in Fig. 3. This difference can be explained by the fact that the model used for the simulations remains a first-order approximation and strongly relies on the measurement of the mirror coating absorption coefficient, which is subject to uncertainties given its smallness, or the radius of curvature of the mirrors. An increase of $170 \mu \mathrm{m}$ of the value of the latter parameter allows to match the model to the experimental data, to be compared to a $\pm 1 \mathrm{~mm}$ manufacturing uncertainty. We thus conclude that the observations are consistent with the model and thus allows the observations to be interpreted satisfactorily here. It must be pointed out that the size of the mirrors limits the maximum mode order.

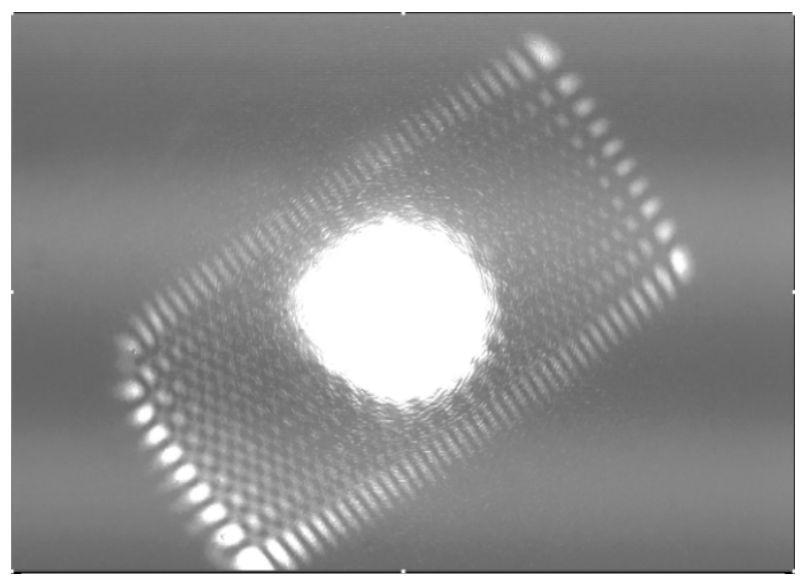

Fig. 4. Superimposition of the resonant high order mode (27.11) and the fundamental mode observed in transmission of $M_{2}$ at an intracavity power of $110 \mathrm{~kW}$. The fundamental mode is saturated to clearly distinguish the larger mode.

It has been observed on different types of FPC geometries that the energy between two resonating modes, in particular $T E M_{n m}$ and TEM $M_{00}$, induces instabilities on the TEM $M_{00}[5,36]$. We indeed observed this effect up to a loss of the lock, see Fig. 5 that shows the intracavity power as function of time. Up to $20 \%$ average power losses are happening due to these degeneracies.

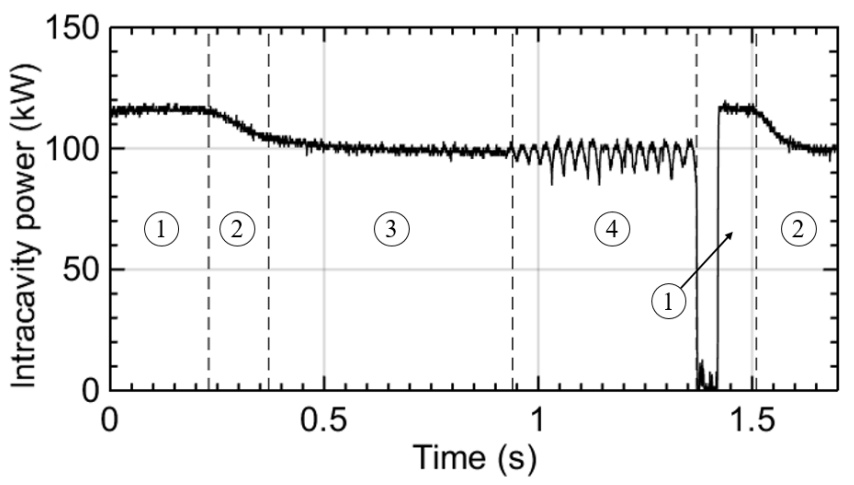

Fig. 5. The curve shows the intracavity power for $1.7 \mathrm{~s}$, measured through $M_{4}$. (1) only fundamental mode, (2) switch to a degenerated mode, (3) stable degenerated region and (4) oscillations due to thermal effects.

The transition time between the fundamental mode (1) and a degenerated regime (2) observed in Fig. 5 is compatible with the transient thermoelastic deformations timescale as computed using $[10,37]$. Oscillations of the intracavity power due to a change of the thermal load on the mirrors are expected [38, 39] and also observed here. They are due to a coupling between 
the dynamics of the thermal diffusion into the substrate and the properties of the electronic feedback loop acting on the laser. The growth of these oscillations irremediably induces a loss of the lock of the system and thus prevents its long term operation at average intracavity power above about $100 \mathrm{~kW}$. Performance goals for X-ray sources based on Compton scattering [20] are thus not fulfilled.

\section{HIGH ORDER MODES SUPPRESSION}

In order to circumvent this limitation, several solutions [12-16, 18,19 ] have been investigated but do not satisfy the requirement of operating a FPC at high average power with high-finesse under high vacuum and in an accelerator environment. We thus propose a new technical solution to comply with these requirements by installing two half-round mirrors with knife edge, so called D-shaped mirrors. As shown on the Figure 1, these mirrors $D_{1}$ and $D_{2}$ are installed on motorized translation stages between $M_{1}$ and $M_{2}$, each on a separate transverse axis. This position has been chosen for practical reasons, indeed the beam is almost parallel between $M_{1}$ and $M_{2}$ and the higher order modes have a transverse size large enough so that they can be easily damped. Besides, it is not possible to put that kind of mirror system near to the other waist due to the proximity of the interaction point. We choose knife edge mirrors to allow placing them in the beam with an angle of about $10^{\circ}$. The reflected part of the beam thus hits the vacuum chamber wall to avoid any spurious reflection or additional heat load on the mirrors or their mounts.

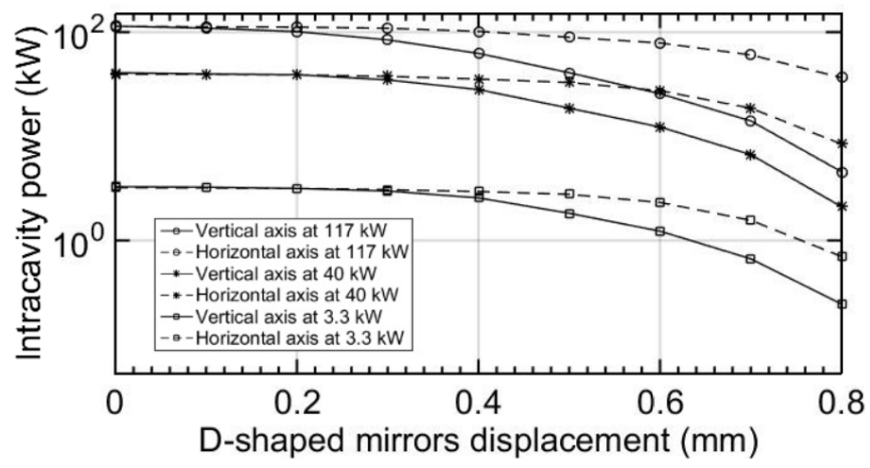

Fig. 6. Experimental measurement of the intracavity power at different D-shaped mirror positions for both vertical and horizontal axes and at several input power values. The numerical value of the displacement increases while the mirrors are getting closer to the optical axis.

It is observed on Figure 6 that, as expected, power losses appear if $D_{1}$ and $D_{2}$ are moved too close to the fundamental mode beam. Several comments could be made from this test. First of all, as expected, there exist a position, in both transverse directions, for which losses are negligible on the fundamental mode. Secondly, it is noticeable that these positions are not the same for both axes and that they depend on the average intracavity power. This is due, at least partly, to the expected different increase of elliptical mode sizes along the two transverse axes induced by thermoelastic deformations [5]. Measured positions and their expected values from calculations are found to be in agreement within $10 \%$. Finally, for beam mode diameters of about $1.8 \mathrm{~mm}$ (horizontal) and $2 \mathrm{~mm}$ (vertical), degenerate high order modes were observed if D-shape mirrors are brought further than $\approx 400 \mu \mathrm{m}$ from these positions. This ensures that there is a comfortable range of D-shape mirrors positions that ensure both damping of the high order modes and negligible losses on the fundamental mode. Experimentally, these positions are determined by requiring no noticeable variation of the transmitted power which is measured with a relative precision $5 \%$, by means of a power meter. One can thus conservatively estimate the maximum losses induced by the D-shape mirrors by assuming that they solely lead to $5 \%$ decrease of the transmitted power. Taking our measured values for the mirror $M_{1}$ transmissivity (180 ppm) and of the total cavity round-trip loss (316 ppm), we obtain a maximum loss induced by the D-shape mirrors of about $8 \mathrm{ppm}$. A cavity optimally coupled with a total round-trip loss of $8 \mathrm{ppm}$ corresponds to a cavity finesse of approximately 400000 . The method proposed here could thus in principle be used for such very high cavity finesse values.

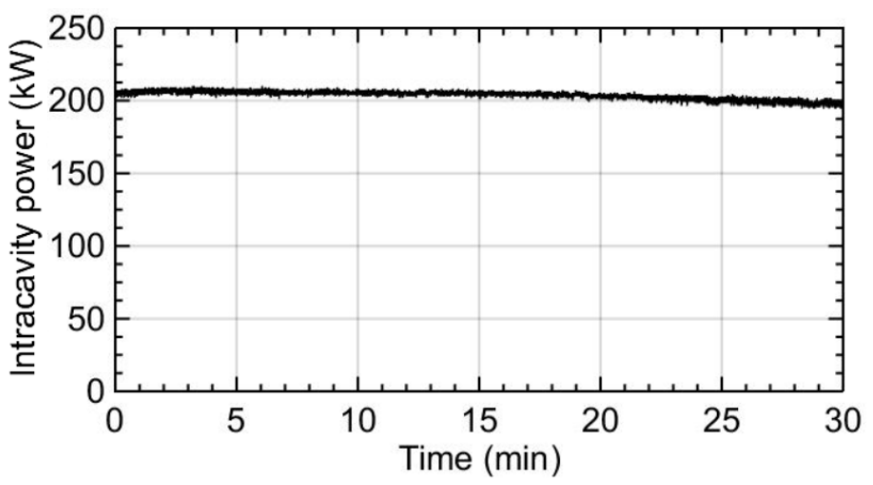

Fig. 7. Laser intracavity power for $30 \mathrm{~min}$, measured by transmission of a cavity mirror.

As a result we have observed clear improvements on the intracavity power stability. In particular, we proceeded a $30 \mathrm{~min}$ run at about $200 \mathrm{~kW}$ for which the evolution of the intracavity power as a function of time is shown on Figure 7. This measurement has been done without any alignment or feedback corrections during the run. A drop of power from $205 \mathrm{~kW}$ to $197 \mathrm{~kW}$ is observed due to thermal stabilization of the vacuum vessel. The initial value of $205 \mathrm{~kW}$ is reached back after further alignment and feedback optimization. This level of intracavity power corresponds to a fluence of approximately $0.04 \mathrm{~J} / \mathrm{cm}^{2}$ on the cavity mirror coatings which is well below damage threshold. We further inspected mirror surfaces with an Atomic Force Microscope to check that no damage has effectively been induced.

\section{CONCLUSION}

In summary, an experimental method to remove high order modes degeneracy in a four-mirror Fabry-Perot cavity is described. A simple model was used to satisfactorily describe the degeneracy observed experimentally, as a function of the intracavity laser beam power, which leads to locking instabilities thus preventing nominal operation of the system. Introduction of D-shape dielectric coated mirrors which are adapted to handle high average power cavity modes because of their non-absorbing properties allowed to damp these high order modes while introducing negligible losses on the fundamental mode. This solution is particularly suitable for a particle accelerator environment typical of Compton back-scattering radiation 
sources. Furthermore, their implementation allows precise and convenient positioning, independent on the two main axes, thus especially suited for all fundamental mode shapes including elliptical beams. We estimated that losses induced by D-shape mirrors on the fundamental modes would fit with cavity finesse of the order of a few hundred thousand. In principle this simple method may thus be used to reach much higher intracavity power. Other processes such as mirror coating damage or degradation may however limit its increase. The former issue can be mitigated by increasing the spot sizes on the mirrors and using $\mathrm{HfO}_{2} / \mathrm{SiO}_{2}$ instead of $\mathrm{Ta}_{2} \mathrm{O}_{5} / \mathrm{SiO}_{2}$ multilayer coatings [40]. The latter issue can also be overcome by using for instance in-situ or ex-situ plasma cleaning as in intense laser beam and synchrotron beam line experiments (see e.g. [41]). In our experiment we indeed observed a decrease of the cavity enhancement factor when increasing the incident laser beam power as it was also reported in [5]. We are presently investigating in detail the origin of such a behavior.

\section{ACKNOWLEDGMENTS}

We are grateful to Dr Jérôme Degallaix for fruitful discussions and his comments on this article.

\section{FUNDING}

This work is supported by the French "Agence Nationale de la Recherche" as part of the program "investing in the future" under reference ANR-10-EQPX-51.

\section{DISCLOSURES}

The authors declare no conflicts of interest.

\section{REFERENCES}

1. L. Federici, G. Giordano, G. Matone, G. Pasquariello, P. G. Picozza, R. Caloi, L. Casano, M. P. De Pascale, M. Mattioli, E. Poldi, C. Schaerf, M. Vanni, P. Pelfer, D. Prosperi, S. Frullani, and B. Girolami, "Backward compton scattering of laser light against high-energy electrons: the ladon photon beam at frascati," II Nuovo Cimento B (1971-1996) 59, 247-256 (1980).

2. L. Casano, A. Marino, G. Matone, M. Roccella, C. Schaerf, and A. Tranquilli, "Production of a beam of polarized and monochromatic gamma rays by compton scattering of laser light against high-energy electrons," LNF-74/60(P) (1974).

3. Z. Huang and R. D. Ruth, "Laser-Electron Storage Ring," Phys. Rev. Lett. 80, 976-979 (1998).

4. I. Pupeza, S. Holzberger, T. Eidam, H. Carstens, D. Esser, J. Weitenberg, P. Rußbüldt, J. Rauschenberger, J. Limpert, T. Udem, A. Tünnermann, T. W. Hänsch, A. Apolonski, F. Krausz, and E. Fill, "Compact high-repetition-rate source of coherent $100 \mathrm{eV}$ radiation," Nat. Photonics 7, 608-612 (2013).

5. H. Carstens, N. Lilienfein, S. Holzberger, C. Jocher, T. Eidam, J. Limpert, A. Tünnermann, J. Weitenberg, D. C. Yost, A. Alghamdi, Z. Alahmed, A. Azzeer, A. Apolonski, E. Fill, F. Krausz, and I. Pupeza, "Megawatt-scale average-power ultrashort pulses in an enhancement cavity," Opt. Lett. 39, 2595 (2014).

6. M. Evans, S. Gras, P. Fritschel, J. Miller, L. Barsotti, D. Martynov, A. Brooks, D. Coyne, R. Abbott, R. X. Adhikari, K. Arai, R. Bork, B. Kells, J. Rollins, N. Smith-Lefebvre, G. Vajente, H. Yamamoto, C. Adams, S. Aston, J. Betzweiser, V. Frolov, A. Mullavey, A. Pele, J. Romie, M. Thomas, K. Thorne, S. Dwyer, K. Izumi, K. Kawabe, D. Sigg, R. Derosa, A. Effler, K. Kokeyama, S. Ballmer, T. J. Massinger, A. Staley, M. Heinze, C. Mueller, H. Grote, R. Ward, E. King, D. Blair, L. Ju, and C. Zhao, "Observation of Parametric Instability in Advanced LIGO," Phys. Rev. Lett. 114 (2015).
7. P. Barriga, M. A. Arain, G. Mueller, C. Zhao, and D. G. Blair, "Optical design of the proposed Australian International Gravitational Observatory," Opt. Express 17, 2149 (2009).

8. A. Simonin, R. Agnello, S. Bechu, J. M. Bernard, C. Blondel, J. P. Boeuf, D. Bresteau, G. Cartry, W. Chaibi, C. Drag, B. P. Duval, H. P. L. d. Esch, G. Fubiani, I. Furno, C. Grand, P. Guittienne, A. Howling, R. Jacquier, C. Marini, and I. Morgal, "Negative ion source development for a photoneutralization based neutral beam system for future fusion reactors," New J. Phys. 18, 125005 (2016).

9. A. L. Bullington, B. T. Lantz, M. M. Fejer, and R. L. Byer, "Modal frequency degeneracy in thermally loaded optical resonators," Appl. Opt. 47, 2840 (2008).

10. P. Hello and J.-Y. Vinet, "Analytical models of thermal aberrations in massive mirrors heated by high power laser beams," J. de Physique 51, 1267-1282 (1990).

11. W. Winkler, R. Schilling, K. Danzmann, J. Mizuno, A. Rüdiger, and K. A. Strain, "Light scattering described in the mode picture," Appl. Opt. 33, 7547 (1994).

12. R. A. Day, G. Vajente, M. Kasprzack, and J. Marque, "Reduction of higher order mode generation in large scale gravitational wave interferometers by central heating residual aberration correction," Phys. Rev. D 87, 082003 (2013).

13. G. Vajente, "In situ correction of mirror surface to reduce round-trip losses in Fabry-Perot cavities," Appl. Opt. 53, 1459-1465 (2014).

14. A. B. Matsko, M. V. Poplavskiy, H. Yamamoto, and S. P. Vyatchanin, "Mitigating parametric instability in optical gravitational wave detectors," Phys. Rev. D 93 (2016).

15. C. Pare and P.-A. Belanger, "Custom laser resonators using gradedphase mirrors," IEEE J. Quantum Electron. 28, 355-362 (1992).

16. T. Klaassen, J. de Jong, M. van Exter, and J. P. Woerdman, "Transverse mode coupling in an optical resonator," Opt. Lett. 30, 1959 (2005).

17. N. Lilienfein, H. Carstens, S. Holzberger, C. Jocher, T. Eidam, J. Limpert, A. Tünnermann, A. Apolonski, F. Krausz, and I. Pupeza, "Balancing of thermal lenses in enhancement cavities with transmissive elements," Opt. Lett. 40, 843 (2015).

18. K. Sakaue, M. Washio, S. Araki, M. Fukuda, Y. Honda, N. Terunuma, and J. Urakawa, "Stabilization of burst laser pulse storage in an optical enhancement cavity using a counter propagating mode," Rev. Sci. Instruments 89 (2018).

19. P. Favier, L. Amoudry, K. Cassou, R. Chiche, K. Dupraz, A. Martens, D. Nutarelli, V. Soskov, F. Zomer, A. Courjaud, and L. Serafini, "Optimization of a Fabry-Perot cavity operated in burst mode for Compton scattering experiments," Phys. Rev. Accel. Beams 21, 121601 (2018).

20. P. Walter, A. Variola, F. Zomer, M. Jaquet, and A. Loulergue, "A new high quality X-ray source for Cultural Heritage," Comptes Rendus Physique 10, 676-690 (2009).

21. F. Zomer, Y. Fedala, N. Pavloff, V. Soskov, and A. Variola, "Polarization induced instabilities in external four-mirror Fabry-Perot cavities," Appl. Opt. 48 (2009).

22. K. Dupraz, K. Cassou, A. Martens, and F. Zomer, "The ABCD matrix for parabolic reflectors and its application to astigmatism free four-mirror cavities," Opt. Commun. 353, 178-183 (2015).

23. J. Bonis, R. Chiche, R. Cizeron, M. Cohen, E. Cormier, P. Cornebise, N. Delerue, R. Flaminio, D. Jehanno, F. Labaye, M. Lacroix, R. Marie, B. Mercier, C. Michel, Y. Peinaud, L. Pinard, C. Prevost, V. Soskov, A. Variola, and F. Zomer, "Non-planar four-mirror optical cavity for high intensity gamma ray flux production by pulsed laser beam Compton scattering off GeV-electrons," J. Instrumentation 7, P01017-P01017 (2012).

24. F. Labaye, "Amplification passive d'un laser à fibre optique dans une cavité fabry-perot : application à la production de rayonnement gamma par diffusion compton inverse," Ph.D. thesis (2012).

25. H. Kogelnik and T. Li, "Laser beams and resonators," Appl. Opt. 5, 1550-1567 (1966).

26. N. Lilienfein, C. Hofer, S. Holzberger, C. Matzer, P. Zimmermann, M. Trubetskov, V. Pervak, and I. Pupeza, "Enhancement cavities for few-cycle pulses," Opt. Lett. 42, 271 (2017).

27. H. Carstens, S. Holzberger, J. Kaster, J. Weitenberg, V. Pervak, 
A. Apolonski, E. Fill, F. Krausz, and I. Pupeza, "Large-mode enhancement cavities," Opt. Express 21, 11606 (2013).

28. K. Numata, S. Otsuka, M. Ando, and K. Tsubono, "Intrinsic losses in various kinds of fused silica," Class. Quantum Gravity 19, 1697-1702 (2002).

29. C. R. Locke, D. Stuart, E. N. Ivanov, and A. N. Luiten, "A simple technique for accurate and complete characterisation of a Fabry-Perot cavity," Opt. Express 17, 21935 (2009).

30. I. Chaikovska, K. Cassou, R. Chiche, R. Cizeron, P. Cornebise, N. Delerue, D. Jehanno, F. Labaye, R. Marie, A. Martens, Y. Peinaud, V. Soskov, A. Variola, F. Zomer, E. Cormier, J. Lhermite, V. Dolique, R. Flaminio, C. Michel, L. Pinard, B. Sassolas, T. Akagi, S. Araki, Y. Honda, T. Omori, N. Terunuma, J. Urakawa, S. Miyoshi, T. Takahashi, and $\mathrm{H}$. Yoshitama, "High flux circularly polarized gamma beam factory: coupling a Fabry-Perot optical cavity with an electron storage ring," Sci. Reports 6 (2016).

31. R. Drever, J. L. Hall, F. Kowalski, J. Hough, G. Ford, A. Munley, and $\mathrm{H}$. Ward, "Laser phase and frequency stabilization using an optical resonator," Appl. Phys. B 31 pp. 97-105 (1983).

32. R. Jason Jones, J.-C. Diels, J. Jasapara, and W. Rudolph, "Stabilization of the frequency, phase, and repetition rate of an ultra-short pulse train to a Fabry-Perot reference cavity," Opt. Commun. 175, 409-418 (2000).

33. A. C. Boccara, D. Fournier, and J. Badoz, "Thermo-optical spectroscopy: Detection by the "mirage effect",' Appl. Phys. Lett. 36, 130132 (1980).

34. S. Gigan, L. Lopez, N. Treps, A. Maitre, and C. Fabre, "Image transmission through a stable paraxial cavity," Phys. Rev. A 72, 023804 (2005).

35. Siegman and A. E., Lasers (University Science Books, Mill Valley, California, 1986), pp. 690-691.

36. J. Benedikter, T. Hümmer, M. Mader, B. Schlederer, J. Reichel, T. W. Hänsch, and D. Hunger, "Transverse-mode coupling and diffraction loss in tunable Fabry-Perot microcavities," New J. Phys. 17, 053051 (2015).

37. Hello, Patrice and Vinet, Jean-Yves, "Analytical models of transient thermoelastic deformations of mirrors heated by high power $\mathrm{cw}$ laser beams," J. Phys. France 51, 2243-2261 (1990).

38. A. L. Bullington, "Thermal loading and modal frequency degeneracy in optical resonators for the laser interferometer gravitational-wave observatory (LIGO)," Ph.D. thesis, Stanford University (2009).

39. T. Klaassen, J. d. Jong, M. v. Exter, and J. P. Woerdman, "Transverse mode coupling in an optical resonator," Opt. Lett. 30, 1959-1961 (2005).

40. L. Gallais and M. Commandré, "Laser-induced damage thresholds of bulk and coating optical materials at $1030 \mathrm{~nm}, 500$ fs," Appl. Opt. 53, A186 (2014).

41. E. Gubbini, U. Eichmann, V. Karpov, H. Schonnagel, M. Kalachnikov, F. Eggenstein, and G. Reichardt, "Decontamination of cpa diffraction gratings," in Summaries of Papers Presented at the Lasers and Electro-Optics. CLEO'02. Technical Diges, (IEEE, 2002), pp. 337-vol. 